\title{
BIM-BASED SPACE MANAGEMENT SYSTEM FOR OPERATION AND MAINTENANCE PHASE IN EDUCATIONAL OFFICE BUILDINGS
}

\author{
Guofeng $\mathrm{MA}^{1}$, Xue SONG${ }^{*}$, Shanshan SHANG ${ }^{2}$ \\ ${ }^{1}$ Department of Construction Management and Real Estate, Tongji University, Shanghai, P.R China \\ ${ }^{2}$ International Business Administration Academic, Shanghai International Studies University, Shanghai, P.R China
}

Received 31 March 2019; accepted 24 July 2019

\begin{abstract}
Lists and floor plans have been widely adopted as space management tools for educational office buildings. However, the two-dimensional floor plans fail to present the indoor complexity, which hinders users from intuitively observing the indoor equipment arrangements and adapting to the indoor environment within a short time. Meanwhile, insufficient research has been conducted on space management tools regarding building indoor navigation. A Building Information Modeling Space Management (BIMSM) system was proposed in this study based on BIM. This system is comprised of two components, i.e. indoor space allocation management and indoor path navigation. The real-time space usage can be queried and user demands may be matched with available space by applying the Space Usage Analysis (SUA) theory. After the establishment of indoor maps, an improved $\mathrm{A}^{\star}$ algorithm is used to provide smooth navigation paths, and the visualization of such paths can be provided in mobile terminals. The BIMSM system was applied in an office building in a university in Shanghai, China. In this case study, the overall user satisfaction reached $91.6 \%$ by greatly reducing space arrangement failures. The time indoor navigation took outperformed that based on the traditional $\mathrm{A}^{\star}$ algorithm, with the search efficiency increasing $5.28 \%$.
\end{abstract}

Keywords: building information modeling (BIM), space management (SM), space usage analysis (SUA), visualization, $\mathrm{A}^{\star}$ algorithm, indoor navigation.

\section{Introduction}

Space management refers to the reasonable allocation and usage of space which improves space utilization to the greatest extent in terms of use frequency and occupancy (Abdullah, Ali, \& Sipan, 2012). This provides people with better work and living environments. Space allocation is an important component in space management (PérezLombard, Ortiz, \& Pout, 2008). More users are willing to check indoor and outdoor information of a building, as well as room usage via space management system. Such information consists of indoor equipment arrangement and decorations, convenience of outdoor paths, and the identity of other users in a shared room. In reality, simple lists and two-dimensional floor plans are used to show information of rooms in educational office buildings, but the lists lack details in terms of equipment layout, indoor and outdoor environments, and room locations, which leads to inappropriate matches and user discontent. Despite that the floor plans overcome such flaws by showing more spatial information, they still cause troubles for us- ers to understand floor plans for all the rooms as well as check indoor equipment and surroundings of the rooms. For instance, when a comfortably decorated environment is needed for activities, it is difficult for users to read such information based solely on the two-dimensional plans. In such circumstances, mismatches may be caused, leading to lower space utilization. Therefore, effective measures should be taken to visually manage the indoor spatial information.

Many people have been aware of the importance of indoor navigation as building structures become increasingly complicated. In a massive mall, customers may fail to understand the simplified maps and locate entrances and exits promptly, and they get lost occasionally. The existing navigation techniques can only provide accurate satellite data outdoors, but may be easily interfered by the building structures (Yan, 2018). The development of indoor navigation techniques, on the other hand, is only on its initial step, with the searches being time-consuming

${ }^{*}$ Corresponding author. E-mail: songxuexs@hotmail.com 
and with poor quality. It is thus worthwhile to find a better approach for this purpose.

The space usage analysis (SUA) method enables the automatic matching between user activities and available space according to their demands (Kim \& Fischer, 2014a; Kim, Rajagopal, Fischer, \& Kam, 2013), this method effectively helps architects examine the space usage in the planning and design phases of a building, i.e. whether certain space is occupied, and take measures timely to eliminate inappropriate use of space. Manual and automatic computer analysis of space usage are included in SUA methods. The manual approach is always time-consuming and error-prone in terms of the expected results of space utilization, whereas the automatic method lacks details of user space demands, such as room area, indoor and outdoor environments. This method is more applied to the planning and design phases of a building instead of the operation and maintenance (O\&M) phase (Zijlstra, Mobach, Van Der Schans, \& Hagedoorn, 2014). However, the O\&M phase is the longest period in the life cycle of a building, its space management is also critical. Managers may improve their work performance by using information readable by standard machines in BIM, such as 3D visualization and roaming, which can be used to present spatial information, layout of furniture and equipment (Wu \& Gao, 2015).

The $\mathrm{A}^{*}$ algorithm is a heuristic path searching method which brings in the evaluation function. Unlike the conventional Dijkstra algorithm, the $\mathrm{A}^{\star}$ method intelligently evaluates map nodes in the space and navigates the paths towards the most probable correct direction between two selected points (Rodenberg, Verbree, \& Zlatanova, 2016). However, its effectiveness depends on the function, in that more constraints will appear if more information is contained in the heuristic function. In this case, fewer nodes will be required for the search process whose time will then be saved. As the search algorithm depends on indoor map nodes and the generation of complicated maps take a large amount of time (Freitag, Weyers, \& Kuhle, 2017), the geometry information and other associated parameters within BIM provide necessary context for indoor path navigation. Structure attributes, such as their locations and geometry can be obtained using BIM to support the rapid indoor map formation with abundant semantics and data (Chen, 2017).

The BIMSM system was proposed in this study for space allocation and indoor direction in the O\&M phase of educational office buildings. The Space-Use Type Differentiations (SUTDs) are used to classify user attributes and user activities to optimize the SUA theory (Kim \& Fischer, 2014a), thereby guiding the allocation of available space based on user needs. Rapid navigation of paths indoors is realized using enhanced $\mathrm{A}^{*}$ algorithm. To meet multiple space requirements, the BIMSM system has added information regarding space arrangement, equipment, indoor and outdoor 3D visualization, and the identity of other users in a shared room based on taking into account the fundamental space information and space usage. The system initiates by promoting the efficiency of $\mathrm{A}^{*}$ algorithm for path search and introducing Diagonal distance as the evaluation function, then nodes in the OPEN table can be stored using the binary heap method. The primary objectives of this study include (1) combination of the optimized SUA and BIM technologies to check current space usage, and match user demands and available space; (2) realization of the rapid indoor path navigation using optimized $\mathrm{A}^{\star}$ algorithm; (3) space allocation and visualized management of indoor direction using BIM; (4) development of Apps on mobile devices for real-time space management and indoor direction. An educational office building was taken as an example finally to test effective of the method described in this study, and BIMSM was proved to be useful for serving indoor space management.

\section{Literature review}

\subsection{SUA-based research in space management}

The SUA method is used to estimate the extent of occupancy by users in each room of a building by analyzing space usage (Kim \& Fischer, 2014b; Kim, 2013), including matching user activities and available space, and predicting space utilization. This method has become all the more important for space management (Gibson, 2000).

Architectural planning theory initiated the development of SUA. Cherry (1999) introduced the formula for calculating space utilization of educational buildings, based on which appropriate space could be allocated to corresponding user activities. However, this method cannot be exclusively used for the analysis of space usage, as the formula for space usage it provides fails to quantitively determine the relationship between users, their activities, and space, the results to some extent rely on the professional knowledge of architects and personal experience. This manual analyzing procedure is time-consuming and error-prone. To determine the relationship between user, user activity, and space (Ioannidis, Tzovaras, \& Malavazos, 2012), Pennanen (2004) considered both the amount of user activities and the time of occupancy which was the activity load. A formalized approach for calculating space utilization was proposed to be an effective standard for developing the automated SUA. However, spatial information is still required to be collected manually in this theory, and its priority lies in calculating space utilization instead of an algorithm for mapping user activities to usable space. To address such issues, Kim et al. (2013) developed a framework, namely knowledge-based space-use analysis (KSUA), which involved space, user and user activity. This framework can help estimate space usage, plan space layouts, and support computer-based SUA. Its flaws include failing to elaborate user activity ontology and lacking proof of feasibility in practical applications. Consequently, Kim and Fischer (2014b) established a general glossary to describe user activities that could be recognized by com- 
puters to improve the design of building space. Although the ontology establishment promoted the development of SUA, the matching between user activity and usable space has still not been mentioned. Based on such a theory, Kim and Fischer (2014a) proposed a method for automating SUA. User activities can be described via selection and combination of SUTDs based on space classification for activities with the help of SUTDs. This approach enables the automatic generation of activity-space pairs (i.e. matching between user activity and free space). Chen and Kim (2017) optimized the current SUTDs and added the seventh space type differentiation, which was whether flexible space could be used. The current SUTDs originate from user activity attributes defined by specialists, which has limitations because of their subjectivity. Also, users tend to consider multiple factors including available space, surrounding environment, interior equipment, etc. during the O\&M phase in real-life practices.

To sum up, the SUA theory was developed from manual analysis to automated computational applications, enabling the matching of available space and prediction of space utilization, but considerations were insufficient for user space demands while emphasizing solely the number of users within a room and whether the required equipment existed in the room, without explaining the details of the equipment (Yan, 2018). It works well for the design phase of building construction, but poorly for the O\&M phase. Space management in the O\&M phase will be the priority in future research.

\subsection{Indoor path planning and navigation research}

The path planning algorithm is the primary element in the research of indoor space navigation, which is applied for user path search and deriving reasonable paths in various conditions. Structures have been increasingly complicated for different purposes in massive buildings, leading to difficulties in indoor space navigation. Current commonly used shortest-path methods for indoor path navigation include Dijkstra algorithm, Ant Colony Algorithm, and A* algorithm, etc. (Ferguson \& Stentz, 2006; Sun, Druzdzel, \& Yuan, 2007).

Liu, S. Ma, and S. Ma (2011) designed a framework to calculate the dynamic shortest path for vehicle navigation. The Dijkstra algorithm was improved based on the average speed of vehicles on different parts of the road network in different time periods, and the shortest path with its time for driving could then be derived. Manlises, Yumang, Marcelo, Adriano, and Reyes (2016) used indoor image editing techniques and the Dijkstra method to plan the shortest path for blind people. The Dijkstra algorithm is commonly used as the most classical approach for calculating the shortest path, but may cause large amounts of useless search of paths. Researches have shown that the $\mathrm{A}^{*}$ method is more time-saving than the Dijkstra one however the length of path varies between the same starting point and destination (Xu, 2007). The Ant Colony Algorithm, as the representative of intelligent algorithms, might reduce the diversity of ant colonies by applying the positive feedback mechanism. This weakens its global search ability to acquire the best path. The $\mathrm{A}^{*}$ method is thus more advantageous in path search.

Despite that the $\mathrm{A}^{*}$ algorithm is better than the Dijkstra one in terms of search time, Wang, Dong, and Zhang (2018) stated that still significant amount of time and space could be wasted during its planning. To solve its problem of containing excess nodes and adjust directions at turnings, Wang (2012) proposed a method for the calculation of turning points, rotation of robots and the smallest angle of doing so. Botea, Müller, and Schaeffer (2004) applied the $\mathrm{A}^{*}$ algorithm on the first hierarchy of the indoor map and optimized the heuristic function based on the characteristics of the map hierarchies.

It can then be concluded that although the $\mathrm{A}^{\star}$ algorithm has advantages in calculating the shortest path indoors, it has flaws including time-consuming search, inefficiency, insufficient smoothness of paths, and high reliance of search performance on the choice of heuristic functions. It is thus necessary to consider such problems for efficient indoor direction.

\subsection{BIM-based research in space management}

BIM provides a platform that records all the information regarding the life cycle of a building in detail, including graphic, non-graphic information, and 3D visual information. Such information solves the problem of abstracting traditional operation management and provides managers with an intuitive and clear visualization model which helps them deepen their understanding of construction projects (Hu, Tian, Li, \& Zhang, 2018). Due to the powerful functions and potential advantages, BIM has been studied to be applied to building space management.

Hallberg and Tarandi (2011) designed the life cycle O\&M framework concerning building space management, equipment maintenance analysis, and stock management, illustrating systematically the huge advantage of BIM throughout the life cycle of a building. Becerik-Gerber, Jazizadeh, $\mathrm{Li}$, and Calis (2011) argued that a space management platform can be provided to managers by BIM, in which information regarding spatial attributes and $3 \mathrm{D}$ visualization facilitates recognition of unused rooms, thereby simplifying space allocation and analysis. Choi, Lee, Park, Cho, and Kim (2014) improved work space planning via path analysis based on information provided by BIM. Nepal, Staub-French, and Pottinger (2012) described the process of extracting and checking specific spatial information with BIM and developed a platform for such purpose. Atazadeh, Kalantari, Rajabifard, Ho, and Champion (2017) investigated a sequence of data elements for managing complex ownership spaces and expanded the open data model regarding BIM. A multi-floor building model can be illustrated, and 3D visualization and data visualization in BIM can be well utilized. The Flexible Space Subdivision Framework (FSS) (Diakité \& Zlatanova, 2018) was proposed to be applied in BIM for office buildings. This 
framework can identify indoor space required for automatic navigation and help users adapt to complex indoor space environment rapidly, navigate visitors both outdoor and indoor, and locate required equipment. The User Activity Simulation and Evaluation Method (UASEM) (W. Shen, Q. Shen, \& Sun, 2012) based on BIM simulates daily activities, particularly regarding space planning in a new building, which promotes interaction between architects and users and strengthens user understandings of the design proposal applied to the new building.

As can be concluded from the above review, research on space management with respect to BIM already conducted focuses more on space arrangement and planning while less on space allocation and indoor direction. Meanwhile, BIM provides 3D space-sharing model and visualization management and lays a solid foundation for coordination and decision-making in the life cycle of a building. The realization of functions in such a model is thus practical.

\section{Space allocation research based on improved SUA}

\subsection{Extended SUTDs}

To enable room selection based on user demands and reasonable allocation in line with BIM-based space management, this study added the consideration of user space demands on the SUTDs proposed by Kim and Fischer (2014a) based on literature review and case studies. Three components were considered after this improvement.

SUTD1: User activities can be categorized as either regular or irregular in a specific building. Regular activities are those that happen frequently as we normally perceive, whereas irregular activities happen less frequently. For instance, in educational office buildings, daily office activities are considered regular, and evening parties and social gatherings between departments which occur less are considered irregular. In this study, it is assumed that regular activities happen in private or shared offices and irregular ones may happen in conference rooms or meeting rooms as shown in Figure 1. According to Office Building Codes in China, a conference room refers to one with a size between 30 and $60 \mathrm{~m}^{2}$, whereas a meeting room refers to one with a size typically between 15 and $30 \mathrm{~m}^{2}$.

SUTD2: Users can be classified into key users and ordinary users, depending on their positions and contributions.

SUTD3: User space selection can be based on personal space preference and minimum space requirements. A portion of users are assumed to share relatively small office areas in a building, such that questionnaires are used to determine minimum requirements and personal preferences in this study. Key and ordinary users in an educational building covering professors, associate professors, lecturers, and administrative staff were invited to fill in questionnaires. Questions were about ideal space requirements and the space specified by educational. 120 questionnaires were distributed and all of them were appropriately responded. Personal preference outweighed minimum space requirement for 109 of the 120 staff, indicating that for most people, these two demands were different, such that it was significant to treat them separately. As a result, it is assumed in this research that the personal preference is a more significant factor. Additionally, key users were assumed to own privileges in that rooms can be selected either according to their personal preferences for appropriate matching between user activity and usable space or based on minimum space requirements. Ordinary users, on the other hand, are assigned with spaces often based on minimum space requirements. This prevents ordinary users from privately occupying large spaces.

As shown in Figure 2, users take into account many factors when choosing spaces based on summary from literature review and questionnaires. Personal preference considerations include room size, room location (i.e. layout of next-door room, distance from room to entrance and exit, lightening, etc.), layout of indoor equipment, and number of users in a room (i.e. if shared, information regarding colleagues sharing the room is needed), among which the most popular options are room location and colleague identity based on questionnaire results. These demands can be met by showing users the plan of the space, outdoor and indoor 3D roaming functions.

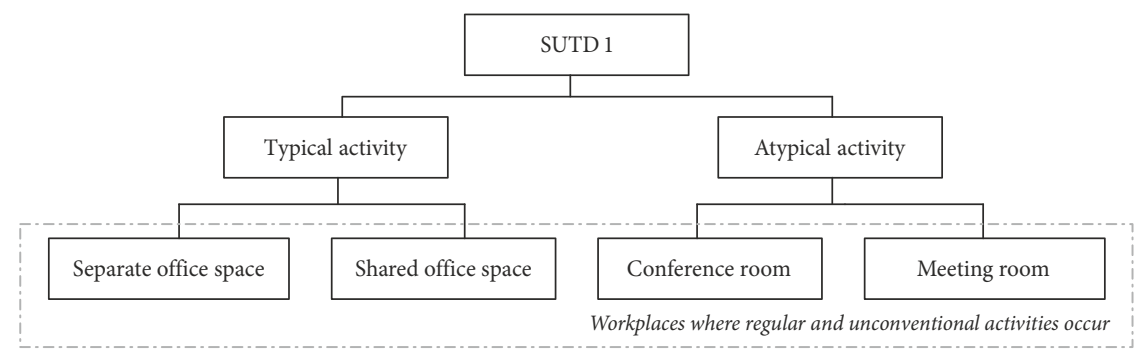

Figure 1. Functional spaces to be selected 


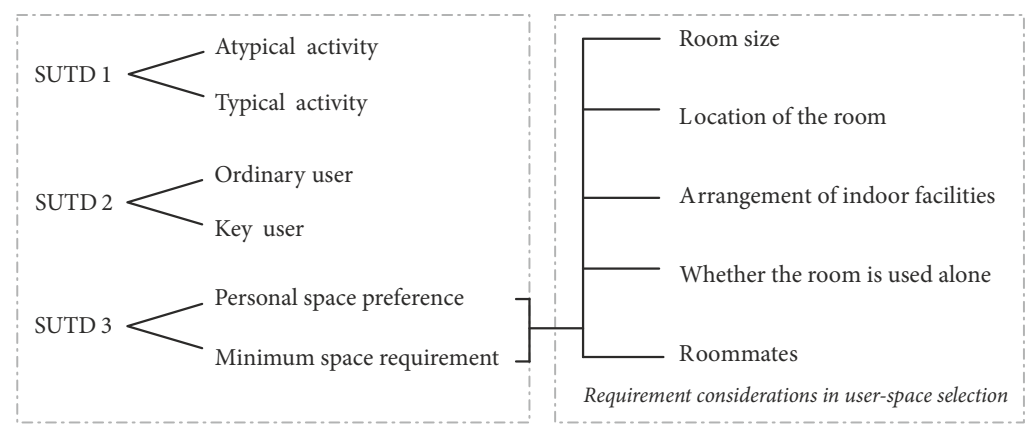

Figure 2. Type of user activity space analysis

\subsection{A method for generating activity-space pairs (user activity and space pair generated after matching)}

The process of matching between user activities and available space in educational office buildings is described as follows. This process requires certain prerequisites and the allocation approach is presented in Figure 3, including 3 phases.

\section{(1) Selection of space type}

Users need to specify their needs for type of space prior to allocation, i.e. regular activities in offices or irregular ones in conference rooms. Offices and conference rooms are open to both key users and ordinary ones.

\section{(2) Selection of space requirements}

Users need to choose appropriate spaces based on their preferences after selecting the type of space. For key users, when their personal preferences are identical to the minimum requirement, personal preferences will be the prin- ciple for allocating rooms, and when they disagree, users may adopt any option. For ordinary users, no matter if their preferences accord with the minimum requirement, the minimum requirement option should be adopted. This procedure is described in Figure 4. For example, key users may choose single offices or shared ones, and choose large or medium/small conference rooms considering their particularity. Allocation of spaces for ordinary users, on the other hand, refer to the lowest standards, i.e. shared offices and medium/small conference rooms.

(3) Pairing user activity and available space

After the above two phases, space that meets all requirements will be screened for further check and selection. Space will continually be examined until if the first appropriate office or conference room available is identified. After this, the room information will be stored in the computer for reasonable matching between user activities and space.

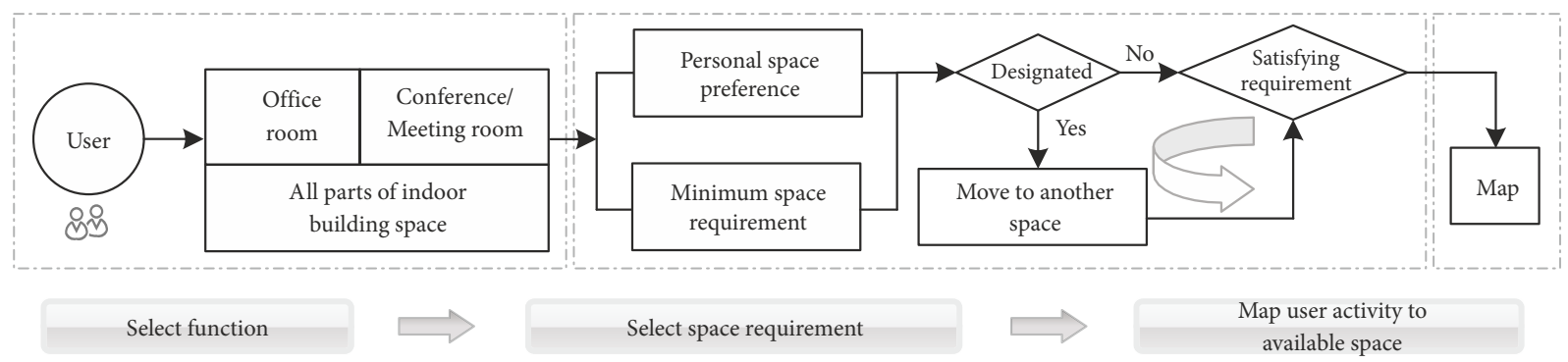

Figure 3. Activity-space pair allocation phase

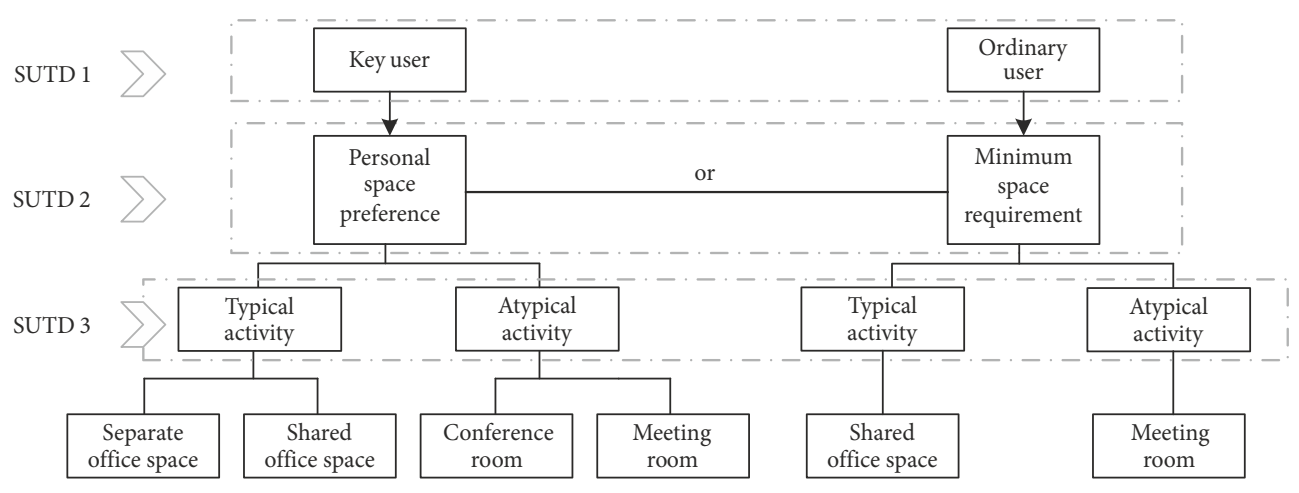

Figure 4. Space requirements based on user types 


\section{Indoor path navigation based on optimized $\mathrm{A}^{*}$ algorithm}

\subsection{Establishment of multi-floor indoor grid maps}

The approaches of indoor mapping include visibility graph method, grid map method, and topological method. The visibility graph method connects the vertices of polygon obstacles with the starting point and the destination using visible straight lines. These straight lines and polygon edges are then given weights respectively. The grid map method divides the indoor map into fixed-size grids, and the grids are attached with specific values to indicate different areas. In a topological map, rooms and space are conceptualized as points, whereas doors connecting them are considered as edges. The conceptualized points and edges will then be given values to form indoor maps. These three approaches vary in characteristics but the visibility graph method is complex in terms of time and space. Graphs with extremely complicated structures will be generated in indoor space with complex environments, thereby causing inefficient navigation. In comparison, the grid maps can determine appropriate grid sizes and adjust grid resolution based on practical needs. They are competitive in terms of flexibility and control of accuracy. The grid map method is thus chosen to form indoor maps, and a BIM-based automatic indoor grid map generation method is designed.

Indoor space can be classified as horizontal or vertical. Horizontal space consists of the space in each floor as well as building structures including walls, pillars, and doors, while vertical space is comprised of elevator shafts and staircases between the floors. The grid maps are formed by first assigning values to the structures in each floor to form separate grid maps, then connecting them by nodes between the floors, i.e. elevator shafts and staircases. A grid map is described as:

$$
E=\left\{g_{i j} \mid g_{i j}=-n \text { or } 0 \text { or } n, n, i, j \in N^{+}\right\} .
$$

In this formula, $g_{i j}$ represents a single grid within a grid map, in which $i$ is the row and $j$ is the column. There are three ways to determine the grid value: when $g_{i j}=$ 0 , the barriers between floors are represented, including walls and pillars. These grids are colored black in the map; when $g_{i j}=n$, the accessible space in a floor is represented, such as doors and corridors, in which $\mathrm{n}$ is the floor number. These grids are colored white in the map; when $g_{i j}=-n$, the grids represent vertical structures including staircases and elevator shafts, they are colored white in the map because they are also accessible.

\subsection{Optimization and improvement of the $\mathrm{A}^{\star}$ algorithm}

The $\mathrm{A}^{*}$ algorithm is among the most efficient methods for path search whose principle is to introduce the evaluation function to search each node, and continue the search process from the last point where the accumulated path length is the minimum. The function is:

$$
F(n)=G(n)+H(n),
$$

where $F(n)$ is the evaluation function that represents node $n$, which is the cost it takes from the beginning of the path to the destination via node $n ; G(n)$ and $H(n)$ represent the first and second half of the cost of path divided by this node. Two state tables are required in the $\mathrm{A}^{*}$ algorithm, i.e. and OPEN table and a CLOSED table.

However, the minimum cost that $\mathrm{A}^{\star}$ algorithm desires is the primary reason of its inefficiency. As all successive nodes after the current one are examined for a single search, and if a node does not exist in the OPEN table, it will be added into the table, otherwise the $G$ value will be used to determine whether the planned path is the optimum solution between the current node and the successive one. The heuristic function will be invoked that calculates the $F$ value, after which the node with the least cost is selected for the next round of calculation, the cost is repeatedly calculated, and the OPEN table needs to store a large amount of node information, which leads to low path search efficiency and huge time consumption. This section of this paper attempts to solve such problem and make improvements where necessary, including the selection of heuristic function and the optimization of search speed in the OPEN table.

An appropriate heuristic function is needed to reduce the search period of nodes to guarantee a highly efficient calculation. $G(n)$ is determined value as known from Eqn (2), such that $H(n)$ is the decisive factor in determining the heuristic function $F(n)$. Common ways of calculation $H(n)$ include Manhattan distance, Diagonal distance, and Euclidean distance. The time they take, the number of nodes they search, and the respective path lengths were analyzed in this study, several experimental results are shown in Table 1 below. The path unfeasible for the Diagonal approach was replaced with the Manhattan one. The Diagonal distance was tested to be the most time-saving option, such that it was chosen as the heuristic function for indoor path search.

To accelerate the search process in OPEN Table, the binary heap (Weiss, 1997) was applied to store nodes in the Table. Its efficiency of inserting and removing nodes is proved to be 2 to 3 times higher than that of the ordinary sorting algorithm, which can be used to tackle the time-consuming issue in $\mathrm{A}^{\star}$ algorithm. When a node is inserted using the binary heap method, it is first placed

Table 1. Result comparison of $\mathrm{A}^{\star}$ algorithm using different heuristic functions

\begin{tabular}{|l|c|c|c|c|c|}
\hline \multicolumn{1}{|c|}{ Function } & Start & Destination & $\begin{array}{c}\text { Searched } \\
\text { nodes }\end{array}$ & $\begin{array}{c}\text { Path } \\
\text { length }\end{array}$ & $\begin{array}{c}\text { Search } \\
\text { time }\end{array}$ \\
\hline $\begin{array}{l}\text { Manhattan } \\
\text { distance }\end{array}$ & $(2,5)$ & $(19,13)$ & 71 & 21 & 19 \\
\hline $\begin{array}{l}\text { Diagonal } \\
\text { distance }\end{array}$ & $(2,5)$ & $(19,13)$ & 62 & 16 & 15 \\
\hline $\begin{array}{l}\text { Euclidean } \\
\text { distance }\end{array}$ & $(2,5)$ & $(19,13)$ & 89 & 19 & 26 \\
\hline
\end{tabular}


at the end of the array, the medial node is then chosen to be compared with the inserted node (the medial position if aliquant, is rounded up). If the $F$ value is smaller, the two nodes will be switched, this process continues until $F$ becomes larger than the comparison value. For example, if $F$ is 16 when first inserted, and the array becomes:

$$
\begin{array}{llllllll}
9 & 29 & 19 & 33 & 37 & 29 & 23 & 16 .
\end{array}
$$

16 was placed at the 8th position in the array (from the left), such that it is then compared with the 4th one, i.e. 33 . As $16<33$, these two are then switched to form a new array:

$$
\begin{array}{llllllll}
9 & 29 & 19 & 16 & 37 & 29 & 23 & 33 .
\end{array}
$$

The position of the inserted node is now 4 , then as $4 / 2=2$, the value at the second position is compared with the inserted node value. As $16<29$, these two nodes are then exchanged, forming a new array again:

\section{$\begin{array}{llllllll}9 & 16 & 19 & 29 & 37 & 29 & 23 & 33 .\end{array}$}

The current node position is 2 , and as $2 / 2=1$, the value at the first position is then compared with the inserted node value. As $16>9$, the value 16 has thus found the appropriate position, meaning the insertion of value has completed. The Figure for the binary heap is shown in Figure 5.

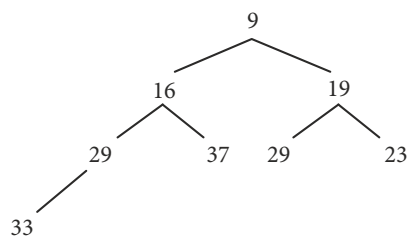

Figure 5. Binary heap after node insertion

The node removed from the OPEN Table is the smallest value in terms of $F$ using the $\mathrm{A}^{*}$ algorithm. The removing process is the reverse that of the insertion. The first node in the array is deleted at first, then the last node in the array is placed at the original first place. The first and second nodes from the left are then compared, if $F$ is smaller than both of them, it is then placed at the right position when the binary heap needs no corrections. If it is larger than either one of them, it is then exchanged with the smaller node. The process repeats itself until no node is left to be compared.

\section{Space management system based on BIM}

\subsection{System development environment}

This chapter describes the development environment and tools for the BIMSM system. Its server is based on Microsoft Windows 10. Autodesk Revit which had the best adaptability was employed for model building. Microsoft Visual Basic 2015 was used for the development using the Application Programming Interface (API). The building model was established and maintained using Autodesk Revit 2018, and the extraction of structures and spatial information was conducted using Visual Basic via Iexter- nal Command. The BIM technology was applied in the BIMSM system for better indoor space management and finer indoor direction.

\subsection{System development process and schematic design}

The BIMSM system was developed for space allocation and navigation using information collected from BIM. The BIM model consists of geometric, parameter, and 3D visualization information. Geometric information is the dimension data of inner building components and groups, including the lengths, widths, and heights of the components as well as room areas which are critical to the function development. For example, when an office is selected, a user may take into account the room space, floor, and surrounding environments, which must be obtained from BIM for diverse space selection. In comparison, parameter information includes the name, number, and condition of indoor equipment.

In terms of space allocation, the BIM model information was used for VB.NET to generate algorithms, acquiring room space, dimension, floor, indoor equipment, to realize BIMSM space allocation development with the help of the improved SUA method as shown in Figure 6.

Space matching of offices and conference rooms is then processed by space preference or minimum requirements based on user attributes while the occupancy conditions can be recorded by the system. Report files of room usage can be generated from the records of occupied rooms, available rooms, and all other indoor space to analyze space utilization. The codes for room space, floor and volume information extraction are listed as follows.

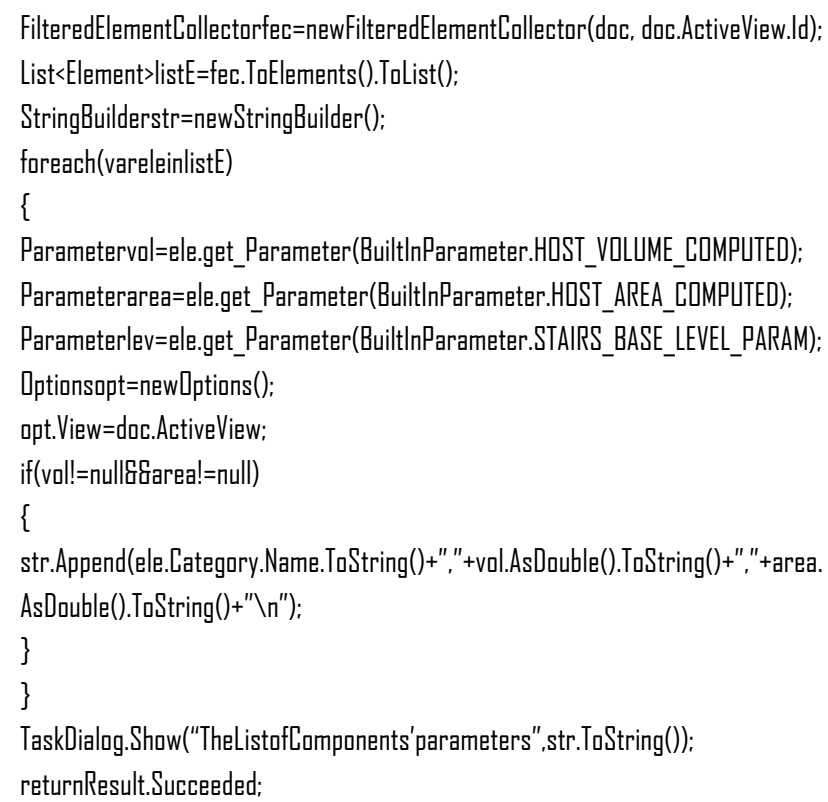

With respect to indoor space navigation, information regarding walls and pillars can be extracted via the API interface. This is combined with the indoor grid partition to automatically generate indoor space maps and guide 
indoor path planning. Grid planes of the number of floors can be first generated by mapping according to the maximum boundary of a building, then walls, pillars, doors, staircases, and elevators can be recognized in BIM. Their position, geometric information, parameter information, and semantic data will then be extracted for mapping all structures on each floor to grid planes. Grid planes on multiple floors will be eventually generated by outputting staircases and elevators based on assigned grids. By using the multiple indoor grid maps, starting point and desti- nation, a planned path will then be generated using the improved $\mathrm{A}^{\star}$ algorithm for indoor direction. The principle of the BIMSM system is described in Figure 7.

\subsection{System modules}

The BIM-based space allocation system consists of indoor space allocation and indoor direction, containing tools for user setup, 3D roaming, and room allocation and checking, and mobile applications.

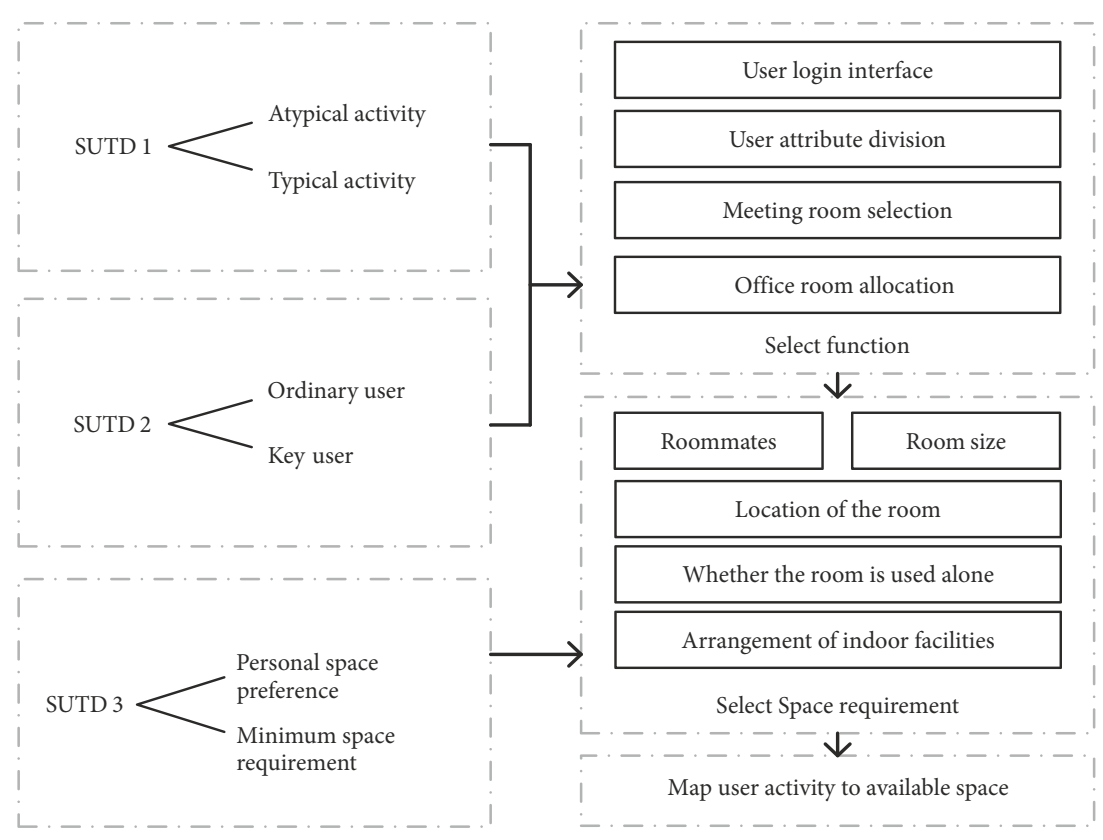

Figure 6. Principle of BIM-based indoor space management system

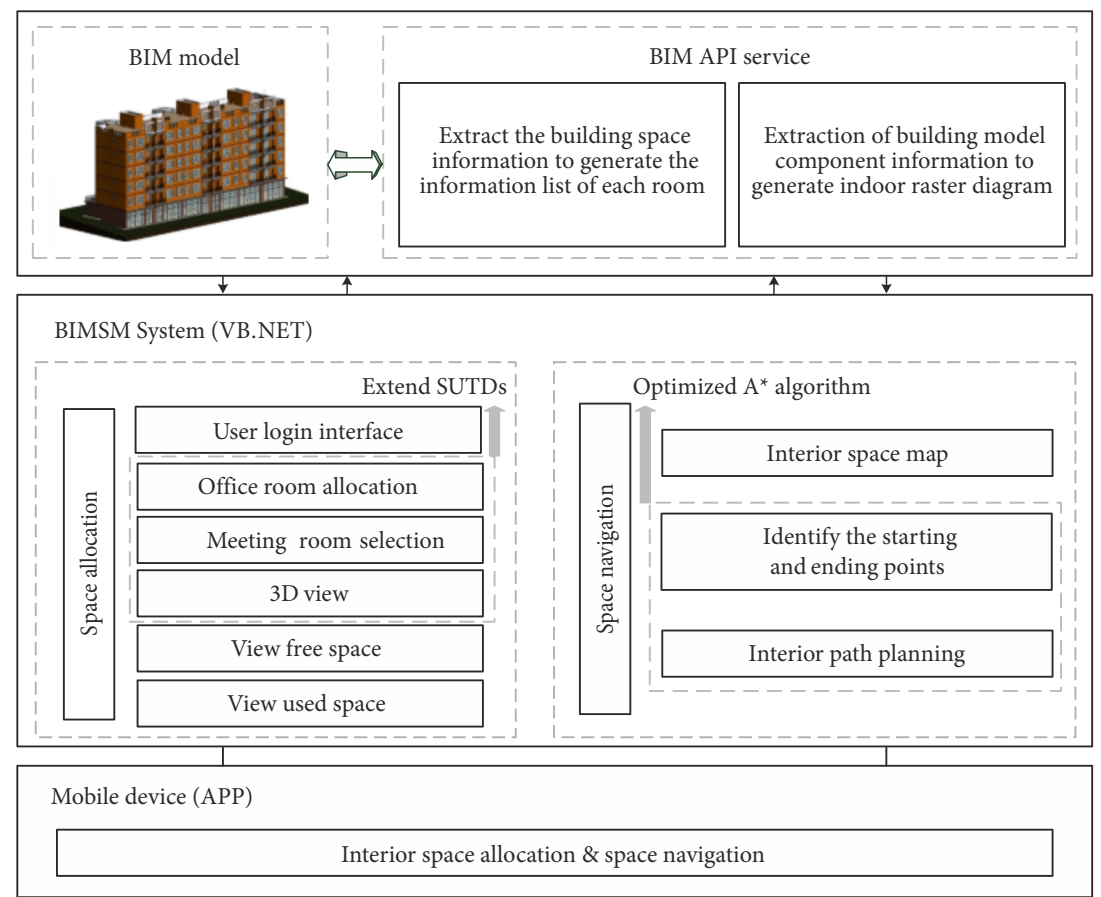

Figure 7. Principle of BIM-based indoor space management system 


\section{(1) User setup module}

Users may login or set room capacities in this module. They may type in their account information and login the space management system. The system automatically recognizes user attributes based on the previous recorded information, and presents the regarding name, age, and position. A browse mode for guests is also set up for them to use indoor direction. In the room capacity setup interface, the average space for various purposes can be prescribed either by fixing the number of people in the room, or by fixing the allocated area per capita, if indivisible, the allowed people in a room is rounded down. The plugin allows the key users to access the setup page, and the results will be shown afterwards for reference.

\section{(2) 3D roaming module}

After the selection of room based on user preference, its location and indoor equipment can be checked using the $3 \mathrm{D}$ roaming module. This function has overcome the problems of traditional space allocation tools to provide intuitive and clear surrounding environment and layout. A $3 \mathrm{D}$ roaming view of a conference room is shown in Figure 8 where mouse sliding can be used in ways of "third-person view, gravitational view, or collision view" to check the indoor layout concerning the number of desks and chairs, room area, the surrounding environment, and its decoration. These functions are usually inapplicable in current space management tools.

(3) Room allocation and reservation module

This module is developed for conference room allocation, booking and cancelling, and checking and extracting information of used and available space. In the conference

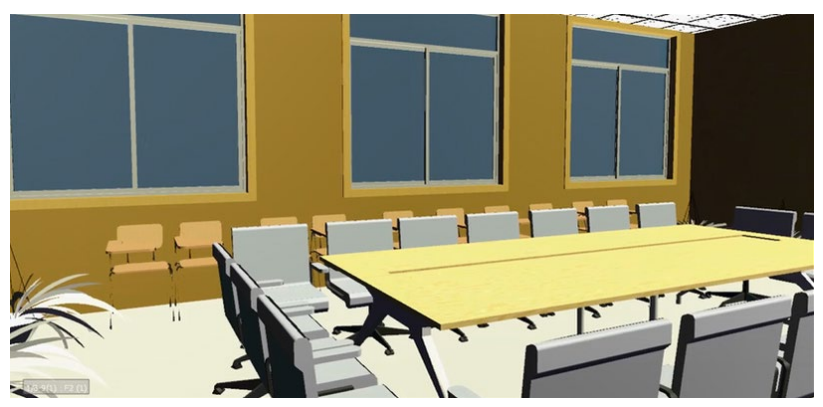

Figure $8.3 \mathrm{D}$ roaming in a conference room

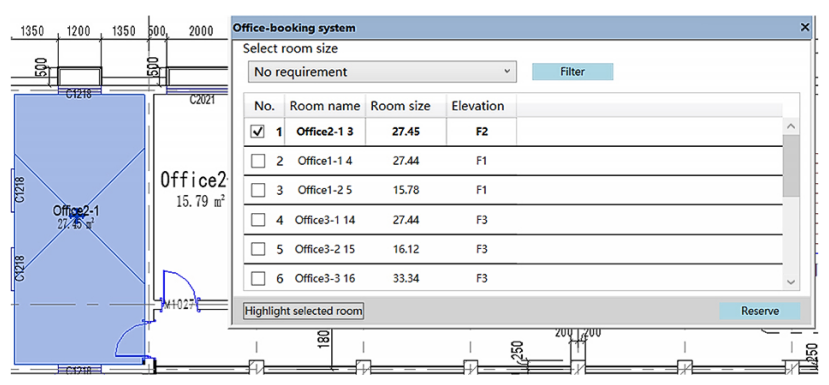

Figure 9. Highlighting selected room room allocation option, rooms can be selected based on user attributes according to SUTDs. All office information can be shown in BIM and users may screen the rooms with their sizes. If rooms of $30 \sim 50 \mathrm{~m} 2$ are filtered, they will be shown in a list, with details including their sequence number, name, size, location and occupancy. The occupancy status includes the number of people using the room and its capacity. For example, (2/5) indicates that 2 persons are using the room which may accommodate 5 users. The current user information may be checked by keep clicking on the number 2. Meanwhile, the selected room can be highlighted in BIM as shown in Figure 9, which facilitates room positioning even if the selected room is not on the current plan. In this process, allocated room information along with its user details may also be checked.

The indoor layout and equipment information of each conference room are provided in the reservation system, including the number and type of desks and chairs, projectors along with other equipment. As shown in Figure 10, the $3 \mathrm{D}$ visualization module may be used to assist room allocation and reservation for desired rooms to be reserved at different times. Multiple conference rooms may be booked based on needs from the users themselves or departments, while the booked ones cannot be rebooked by other people at the same time. The reservation information may be altered or cancelled if necessary. The finalized reservation status will be shown for others' reference.

(4) Indoor space navigation

The navigation module is easy to operate, by simply inputting the starting point and the destination for rapid path plans. Relevant floors can be automatically recognized by the inputs, after which indoor grid maps can be generated for the rapid production of paths using the improved $\mathrm{A}^{*}$ algorithm.

(5) Space management in mobile APPs

Indoor space management and navigation are enabled in mobile applications by typing in the floor, starting point and destination. In Figure 11, the path between Room 3-1 and Room 3-12, and that between Room 3-5 and Room 4-1 are shown staring with the green point, and ending with the yellow one. For paths across multiple floors, they are planned by first connecting the starting point with staircases or elevators. The shortest path connecting them will be selected if multiple staircases or elevators exist.

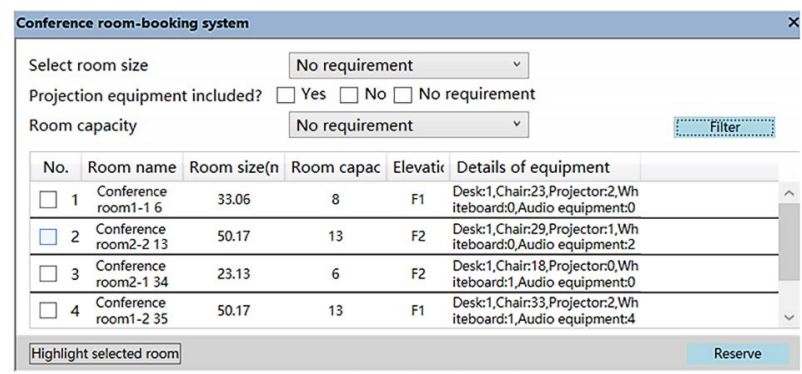

Figure 10. Conference room reservation interface 

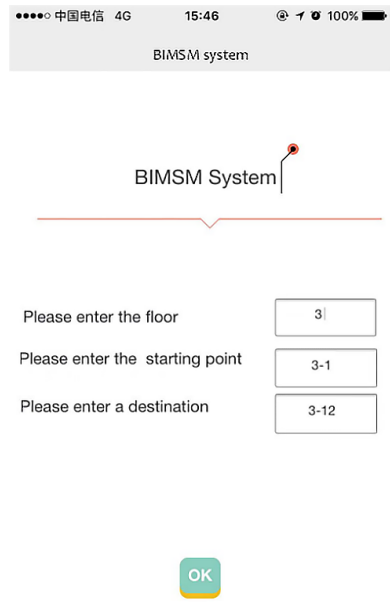
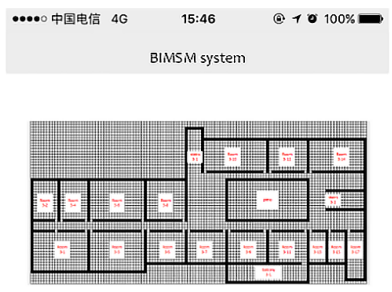

Indoor map

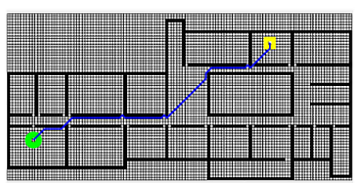

Navigation path
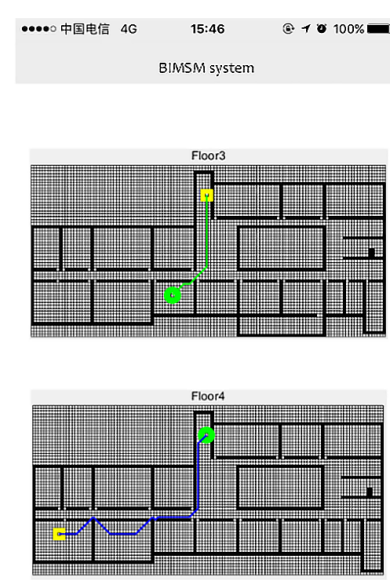

Figure 11. Interface for indoor navigation on mobile APP

\section{System validation}

\subsection{Case study background}

The object of study is a six-floor office building in a university in Shanghai, China, with the outside view shown in Figure 12. Its total building area is $5,400 \mathrm{~m}^{2}$, with brickconcrete structure. This building is equipped with 36 conference rooms and 74 offices. Among the 74 offices, 29 of which are private offices, and the rest of them are shared ones. Secretaries working in the building are responsible for arranging the conference rooms, but troubles may occur using the current space management platforms. Using such platforms, only the occupancy status and floor information can be transferred and no mobile application function is provided. Users need to enter the rooms to check the environment, space, etc., so by the time they return and book the desired conference room, it may already be occupied by other users.

To address the problem of failing to extract indoor space, environment, and layout information, and regular cancelling of reservation due to lack of effective information, the BIMSM system was employed in the building described above. The effectiveness of the system could be evaluated by referring to its cancellation rate, use frequency, efficiency of path planning, and user satisfaction. 53 users participated in the validation of the BIMSM system in the case study, 18 of which were key users. 30 users were then invited for the satisfaction survey.

\subsection{System allocation comparison before and after BIMSM application}

The BIMSM system was applied in this educational office building from September to December 2018. Its operation was compared with that before this study period. The targets for analysis included the frequencies of successful and failed reservations. A successful room reservation indicates the successful use of a room without cancelling after space allocation. This is because during space allocation, leaving the room empty even after successful booking is also a way of reducing space utilization. Consequently, this condition is considered to be a failed reservation. The other scenario for a failed reservation is the immediate cancellation without using the room. Based on such principles, space usage data, i.e. the number of time for failed or successful reservations, before and after the employment of the system were collected on a weekly basis from September to December, 2018 (17 weeks in total) as shown in Figure 13 and Figure 14. It should be noted that during the studied four months, the BIMSM system was not used in the first roughly 9 weeks and the system was then applied in the following roughly 8 weeks.

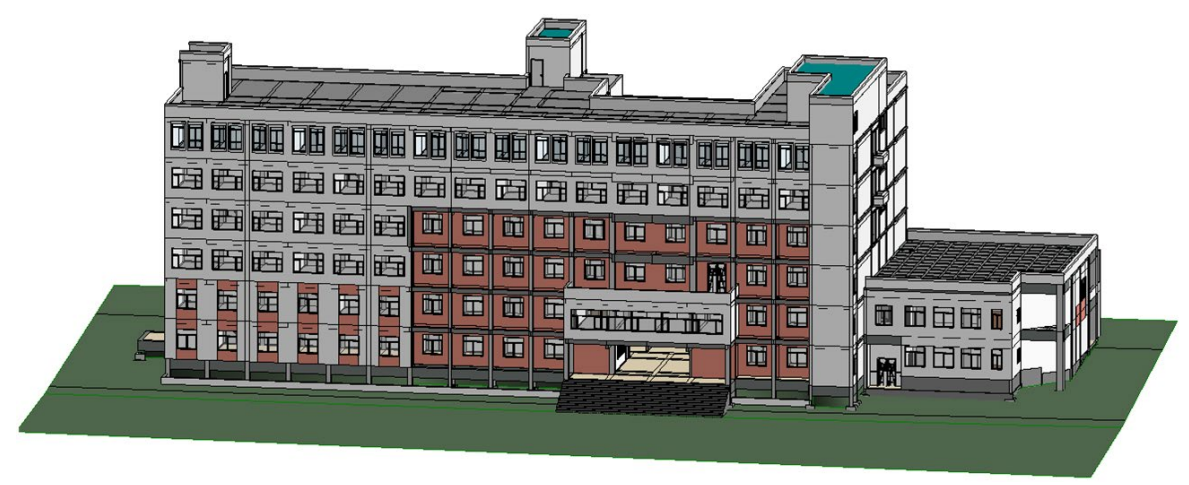

Figure 12. BIM model of a six-floor educational office building in Shanghai, China 


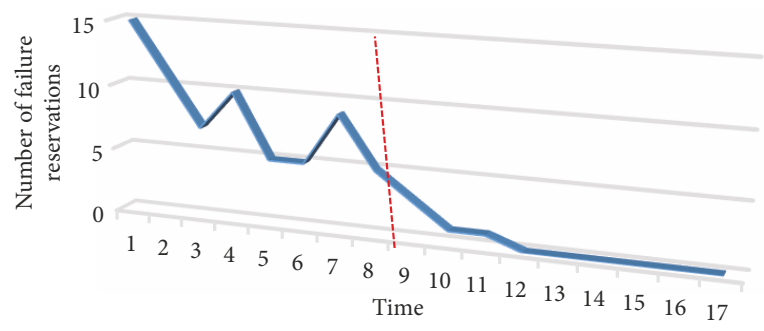

Figure 13. Failure reservation comparison

Figure 13 describes the frequency of failed reservations before and after introducing the BIMSM system, with the horizontal axis being the time, showing the sequence of weeks, and the vertical axis being the failure reservation frequency during the trial period. In the first nine weeks without BIMSM, the number of cancellations remained stable at five times per week. This number decreased in the last three days of Week 9 when the system started working, and it continued to decrease dramatically from Week 10. Cancellations decreased to less than once per week from Week 13 to Week 17. Cancellations apart from human operations almost disappeared, which indicated that the BIMSM system assisted in reducing the cancellation rate in the tested building, and that it could provide users with sufficient spatial and environmental information to make better choices and constrain the waste of space. Figure 14 records the successful reservation in these two periods. Similar to Figure 13, the horizontal axis represents time on a weekly basis, and the vertical axis represents the number of successful reservations. The red line in the figures divides the two scenarios before and after

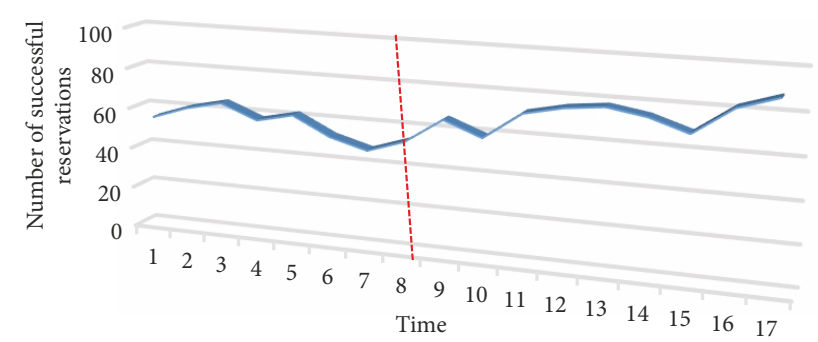

Figure 14. Successful reservation comparison

the employment of the BIMSM system. The successful reservation frequency steadily increased from late Week 9 to Week 17. The lowest frequency after using the system basically equaled the highest frequency before the system's application. By referring to the space allocation data in the past three years from November to December, the successful reservation rate also significantly increased, which proves the improvement of space allocation rate using BIMSM that further helps promote space utilization.

\subsection{Indoor space navigation optimization and comparison}

The indoor path planning was conducted on the $3 \mathrm{rd}$ and 4th floors of the building based on the search time and efficiency in the grid map in BIM. The indoor structures are mapped in BIM to generate grid maps for the two floors shown in Figures 15-18. The conventional and optimized algorithms were compared in this study by recording their search time from the same starting point to the same destination, respectively. Fifty such experiments were conducted, each with varying barriers in between.

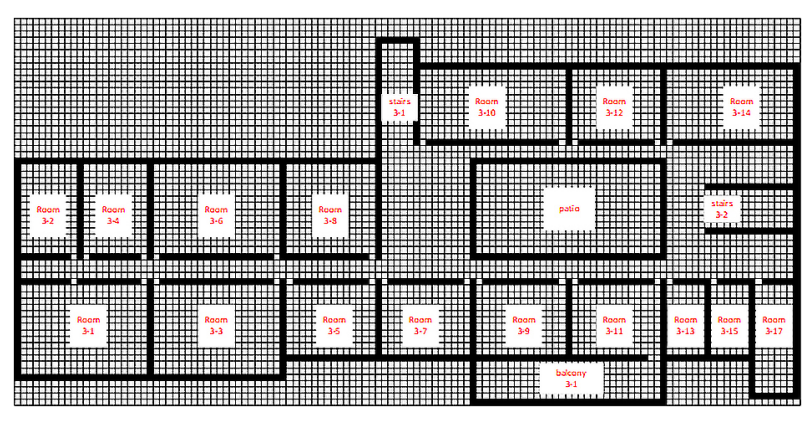

Figure 15. Grid map of the $3^{\text {rd }}$ floor

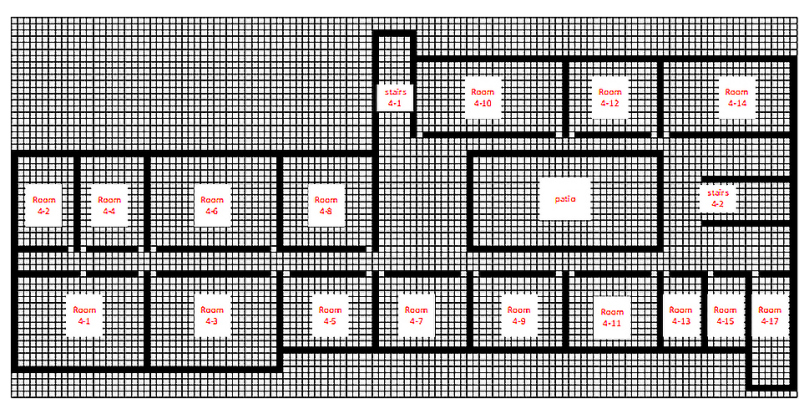

Figure 17. Grid map of the $4^{\text {th }}$ floor

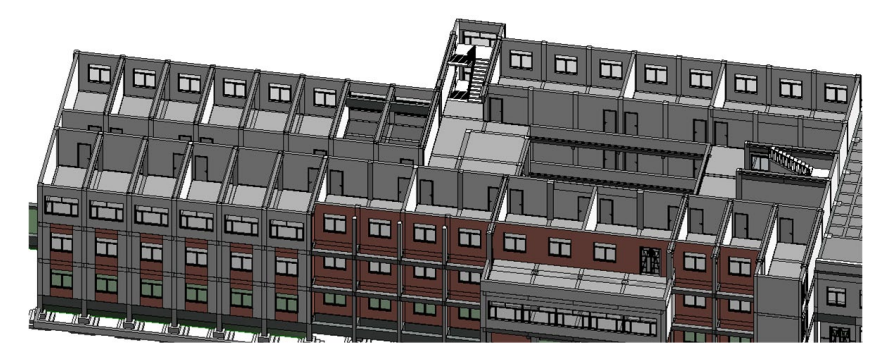

Figure 16. BIM of the $3^{\text {rd }}$ floor

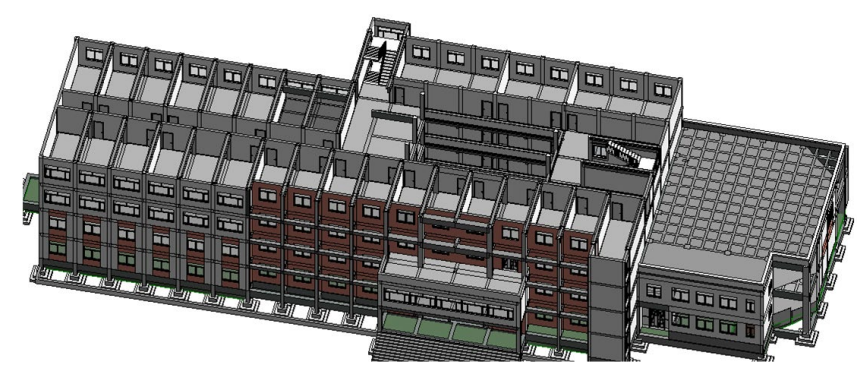

Figure 18. BIM of the $4^{\text {th }}$ floor 
Table 2. Search time comparison before and after $A^{\star}$ algorithm optimization

\begin{tabular}{|l|c|c|c|c|c|c|c|c|c|c|c|}
\hline Number & 1 & 2 & 3 & 4 & 5 & 6 & 7 & 8 & 9 & 10 \\
\hline Time1 & 5.5 & 3.2 & 6.6 & 2.5 & 3.9 & 3.3 & 4.0 & 1.5 & 2.9 & 4.0 \\
\hline Time2 & 5.5 & 3.0 & 6.2 & 2.3 & 3.8 & 3.3 & 3.8 & 1.1 & 2.8 & 3.9 \\
\hline Number & 11 & 12 & 13 & 14 & 15 & 16 & 17 & 18 & 19 & 20 \\
\hline Time1 & 4.2 & 3.5 & 5.1 & 5.4 & 3.6 & 2.2 & 3.4 & 1.8 & 4.5 & 4.1 \\
\hline Time2 & 4.0 & 3.5 & 4.7 & 5.4 & 3.5 & 2.0 & 3.4 & 1.5 & 4.2 & 4.0 \\
\hline Number & 21 & 22 & 23 & 24 & 25 & 26 & 27 & 28 & 29 & 30 \\
\hline Time1 & 2.0 & 1.6 & 2.5 & 5.3 & 1.7 & 2.3 & 3.3 & 3.6 & 4.2 & 5.3 \\
\hline Time2 & 2.0 & 1.5 & 2.2 & 5.1 & 1.3 & 1.9 & 3.3 & 3.4 & 3.9 & 5.1 \\
\hline Number & 31 & 32 & 33 & 34 & 35 & 36 & 37 & 38 & 39 & 40 \\
\hline Time1 & 4.1 & 1.8 & 1.5 & 2.9 & 1.8 & 3.6 & 3.1 & 1.6 & 2.0 & 4.1 \\
\hline Time2 & 4.1 & 1.5 & 1.3 & 2.7 & 1.6 & 3.3 & 3.1 & 1.5 & 1.7 & 4.0 \\
\hline Number & 41 & 42 & 43 & 44 & 45 & 46 & 47 & 48 & 49 & 50 \\
\hline Time1 & 2.2 & 3.1 & 4.2 & 5.1 & 2.8 & 5.6 & 3.5 & 4.1 & 2.7 & 3.6 \\
\hline Time2 & 2.2 & 2.8 & 4.2 & 5.0 & 2.8 & 5.3 & 3.1 & 3.8 & 2.5 & 3.3 \\
\hline
\end{tabular}

The slow conventional search methods normally take a large amount of time because they search the minimum value in the OPEN table. The binary heap method was used in this study to store a lot of nodes that are sequenced for later rapid node insertion and removal, which could save the search time for users. Data for nodes and search time before and after the optimization were prepared in Table 2.

Time 1 in the table denotes the planning time for the conventional $\mathrm{A}^{*}$ algorithm, and time 2 denotes that for the improved one. The search time for the improved method outperformed that for the conventional one in all 50 sets of data. Another 100 sets of tests were performed later to further prove its effectiveness for rapid path guidance. The search time comparison of the 50 sets of data is shown in Figure 19, with the blue line representing the conventional method and the orange one indicating the optimized method. The orange line basically coincides with the blue line or lies beneath it, indicating an improvement of the optimization in node searching, and therefore accelerating indoor direction by $5.28 \%$. For buildings with complicated indoor structures, especially those with many nodes, more time could be saved.

For better judgement of the system's usefulness, questionnaires were distributed and users were invited to mark

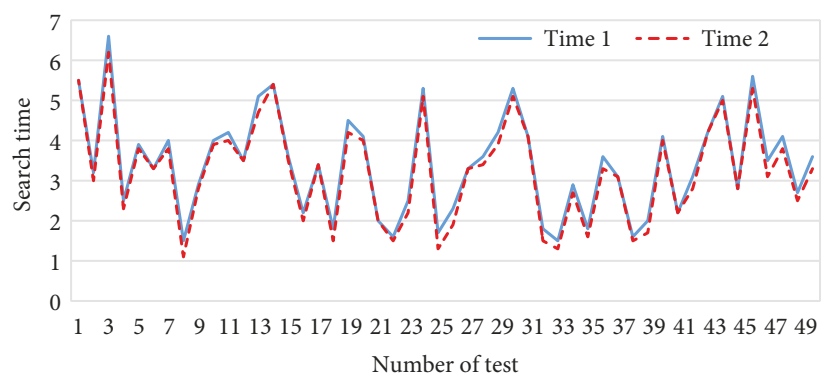

Figure 19. Path searching time comparison before and after optimization the overall system performance based on the Likert Scale method. Among the 30 frequent users throughout the test duration, the satisfaction rate reached $91.6 \%$ and most of them considered the system useful in assisting their work.

\section{Conclusions}

A BIM-based space management system was developed in this research that overcame the disadvantages using traditional management tools. Detailed indoor environmental information was provided with the help of visualized operations in BIM, enabling the combination of SUA and BIM. The space navigation function can help users plan indoor paths in a short time, which fills the gap in current space management tools. Major contributions of this research include:

(1) SUTDs in the SUA method were improved to emphasize user attributes, activity types and demands based on the original seven space usage approaches. Information that users concern including indoor and outdoor space environments were considered along with indoor equipment arrangement, and information of users in a shared room.

(2) The optimized SUA method was successively combined with BIM to timely check the current building usage and map user demands with available space.

(3) The BIM-based automatic indoor grid map generation method was introduced. The search efficiency was promoted using the Diagonal distance evaluation function and the binary heap method to shorten the search time of $\mathrm{A}^{\star}$ algorithm.

(4) Indoor geometric parameters and equipment information could be extracted, processed and exported based on BIM. A secondary development was conducted for automatic data extraction to enable the BIM-based space allocation and visual management of indoor direction. 
The case study indicates that after using the BIMSM system for indoor space allocation, the room cancelling rate declined significantly to approximately zero, proving that sufficient fundamental space and environment information could be provided and indicating the improvement of space utilization. The search time using the BIMSM system for indoor path navigation outperformed that using conventional path search methods by evaluating 100 sets of path planning experiments.

Despite such improvements, flaws still exist, including (1) only educational office buildings were studied in this research without commercial buildings and other office buildings; (2) only key users and ordinary users were taken into account in user classification which normally should be finer to provide diverse choices for different users; (3) in terms of indoor direction, the optimization of the $\mathrm{A}^{*}$ algorithm considers only the heuristic function and the impact of OPEN table on search time, and the path searching efficiency was reduced due to the classification of indoor maps. Finer user attributes and smoother navigation paths should be made possible in future research to promote delicacy of space management.

\section{Acknowledgements}

This research was supported by the National Natural Science Foundation of China (No.71671128).

\section{Funding}

This work was supported by the $<$ National Natural Science Foundation of China $>$ under Grant [number 71671128].

\section{Author contributions}

Guofeng Ma, Xue Song, and Shanshan Shang have conceived the study and were responsible for the design and structure of the paper. Guofeng Ma and Shanshan Shang were responsible for data analysis. Xue Song wrote drafts of the article.

\section{Disclosure statement}

Nothing to declare.

\section{References}

Abdullah, S., Ali, H. M., \& Sipan, I. (2012). Benchmarking space usage in higher education institutes: attaining efficient use. Journal of Techno-Social, 4(1), 11-20.

Atazadeh, B., Kalantari, M., Rajabifard, A., Ho, S., \& Champion, T. (2017). Extending a BIM-based data model to support 3D digital management of complex ownership spaces. International Journal of Geographical Information Science, 31(3), 499522. https://doi.org/10.1080/13658816.2016.1207775

Becerik-Gerber, B., Jazizadeh, F., Li, N., \& Calis, G. (2011). Application areas and data requirements for BIM-enabled facilities management. Journal of Construction Engineering and Management, 138(3), 431-442.

https://doi.org/10.1061/(ASCE)CO.1943-7862.0000433
Botea, A., Müller, M., \& Schaeffer, J. (2004). Near optimal hierarchical path-finding. Journal of Game Development, 1(1), 7-28.

Chen, Y. (2017). BIM-based research on indoor navigation techniques for emergency evacuation. Southwest Petroleum University.

Chen, X. B., \& Kim, T. W. (2017). Automated mapping of user activities onto flexible space in space-use analysis. Journal of Construction Engineering and Management, 143(8), 04017034. https://doi.org/10.1061/(ASCE)CO.1943-7862.0001328

Cherry, E. (1999). Programming for design: From theory to practice. John Wiley \& Sons.

Choi, B., Lee, H.-S., Park, M., Cho, Y. K. \& Kim, H. (2014). Framework for work-space planning using four-dimensional BIM in construction projects. Journal of Construction Engineering Management, 140(9), 04014041.

https://doi.org/10.1061/(ASCE)CO.1943-7862.0000885

Diakité, A. A., \& Zlatanova, S. (2018). Spatial subdivision of complex indoor environments for 3D indoor navigation. International Journal of Geographical Information Science, 32(2), 213-235. https://doi.org/10.1080/13658816.2017.1376066

Ferguson, D. \& Stentz, A. (2006). Using interpolation to improve path planning: The Field $D^{*}$ algorithm. Journal of Field Robotics, 23(2), 79-101. https://doi.org/10.1002/rob.20109

Freitag, S., Weyers, B., \& Kuhlen, T. W. (2017, March). Efficient approximate computation of scene visibility based on navigation meshes and applications for navigation and scene analysis. In 2017 IEEE Symposium on 3D User Interfaces (3DUI) (pp. 134-143). Los Angeles, California, USA. https://doi.org/10.1109/3DUI.2017.7893330

Gibson, V. 2000. Evaluating office space needs and choices. University of Reading.

Hallberg, D., \& Tarandi, V. (2011). On the use of open bim and $4 \mathrm{~d}$ visualisation in a predictive life cycle management system for construction works. ITcon, 16, 445-466.

Hu, Z.-Z., Tian, P.-L., Li, S.-W., \& Zhang, J.-P. (2018). BIM-based integrated delivery technologies for intelligent MEP management in the operation and maintenance phase. Advances in Engineering Software, 115, 1-16.

https://doi.org/10.1016/j.advengsoft.2017.08.007

Ioannidis, D., Tzovaras, D., \& Malavazos, C. (2012, July). Occupancy and business modelling. In The European Conference of Product and Process Modelling (ECPPM 2012). Reykjavik, Island. https://doi.org/10.1201/b12516-145

Kim, T. W. (2013). Predicting space utilization of buildings through integrated and automated analysis of user activities and spaces. Stanford University.

Kim, T. W., Rajagopal, R., Fischer, M., \& Kam, C. (2013). A knowledge-based framework for automated space-use analysis. Automation in Construction, 32, 165-176. https://doi.org/10.1016/j.autcon.2012.08.002

Kim, T. W., \& Fischer, M. (2014a). Automated generation of user activity space pairs in space-use analysis. Journal of Construction Engineering and Management, 140(5), 04014007. https://doi.org/10.1061/(ASCE)CO.1943-7862.0000839

Kim, T. W., \& Fischer, M. (2014b). Ontology for representing building users activities in space-use analysis. Journal of Construction Engineering and Management, 140(8), 04014035. https://doi.org/10.1061/(ASCE)CO.1943-7862.0000881

Liu, J., Ma, S., \& Ma, S. (2011). Dynamic shortest path calculation based on improved Dijkstra algorithm. Journal of Systems Science and Information, 31(6), 1153-1157.

Manlises, C., Yumang, A., Marcelo, M., Adriano, A., \& Reyes, J. (2016, November). Indoor navigation system based on computer vision using CAMShift and $\mathrm{D}^{\star}$ algorithm for visually im- 
paired. In The 6th IEEE International Conference on Control System, Computing and Engineering (ICCSCE 2016) (pp. 481-484). Penang, Malaysia.

https://doi.org/10.1109/ICCSCE.2016.7893623

Nepal, M. P., Staub-French, S., \& Pottinger, R. (2012). Querying a building information model for construction-specific spatial information. Advanced Engineering Informatics, 26(4), 904923. https://doi.org/10.1016/j.aei.2012.08.003

Pennanen, A. (2004). User activity based workspace definition as an instrument for workplace management in multi-user organizations. Helsinki: Haahtelakehitys Oy.

Pérez-Lombard, L., Ortiz, J., \& Pout, C. (2008). A review on buildings energy consumption information. Energy and Buildings, 40(3), 394-398. https://doi.org/10.1016/j.enbuild.2007.03.007

Rodenberg, O., Verbree, E., \& Zlatanova, S. (2016). Indoor A* pathfinding through an octree representation of a point cloud. ISPRS Annals of the Photogrammetry, Remote Sensing and Spatial Information Sciences, 4, 249-255.

https://doi.org/10.5194/isprs-annals-IV-2-W1-249-2016

Shen, W., Shen, Q., \& Sun, Q. (2012). Building Information Modeling-based user activity simulation and evaluation method for improving designer-user communications. Automation in Construction, 21, 148-160.

https://doi.org/10.1016/j.autcon.2011.05.022

Sun, X., Druzdzel, M. J., \& Yuan, C. (2007, January). Dynamic weighting $A^{\star}$ search-based MAP algorithm for Bayesian Networks. In Proceedings of the 20th International Joint Conference on Artificial Intelligence (pp. 2385-2390). Hyderabad, India.
Wang, D. (2012). Robot indoor path planning based on improved $\mathrm{A}^{\star}$ algorithm. Journal of Tsinghua University, 8, 1085-1089.

Wang, W., Dong, P., \& Zhang, F. (2018). The shortest path planning for mobile robots using improved $\mathrm{A}^{*}$ algorithm. Journal of Computer Applications, 38(5), 1523-1526.

Weiss, M. (1997). Data structures and algorithm analysis in C. Pearson.

Wu, H. M. \& Gao, P. (2015). Application of BIM in residential space management of building projects. Project Management Techniques, 13(10), 57-63. https://doi.org/10.23883/IJRTER.2018.4040.GBKO1

$\mathrm{Xu}, \mathrm{Y}$. (2007). Research on shortest path planning in vehicle navigation system. Jilin University.

Yan, L. (2018). Indoor positioning and navigation system design based on Wifi and sensors. Nanjing University of Posts and Telecommunications.

Zijlstra, E., Mobach, M. P., Van Der Schans, C., \& Hagedoorn, M. (2014, June). Facilities planning promoting efficient space use at hospital building. In 13th EuroFM Research Symposium (pp. 366-377). Berlin, Germany. 